\title{
Design of thermolabile bacteriophage repressor mutants by comparative molecular modeling
}

\author{
Arjen Nauta, Bertus van den Burg, Harma Karsens, Gerard Venema*, and Jan Kok \\ Department of Genetics, Groningen Biomolecular Sciences and Biotechnology Institute, University of Groningen, Kerklaan 30, 9751 NN Haren, The \\ Netherlands. ${ }^{\star}$ Corresponding author: (e-mail: g.venema@biol.rug.nl).
}

Received 21 April 1997; accepted 29 August 1997

\begin{abstract}
Comparative molecular modeling was performed with repressor protein Rro of the temperate Lactococcus lactis bacteriophage r1t using the known 3D-structures of related repressors in order to obtain thermolabile derivatives of Rro. Rro residues presumed to stabilize a nonhomologous but structurally conserved hydrophobic pocket, which was shown to be important for thermostability of the Escherichia coli bacteriophage lambda repressor $\mathrm{Cl}$, were randomized. Of the derivatives that exhibited various temperature-sensitive phenotypes, one was shown to hold promise for both fundamental and industrial applications that require the controlled production of (heterologous) proteins in L. lactis.
\end{abstract}

Keywords: protein design, lactococcal phage repressor, temperature-sensitive mutants

The Gram-positive lactic acid bacterium Lactococcus lactis is used extensively in the dairy industry for its ability to acidify milk and its role in the subsequent cheese-ripening process ${ }^{1,2}$. Cheese ripening is highly dependent upon lysis of the lactococcal cells within the cheese matrix, which liberates flavor compounds ${ }^{3}$. As breakdown of lactococcal cell walls is a rather slow process ${ }^{4}$, there is a growing interest for systems that facilitate the controlled overproduction of a cell walllytic enzyme by this organism. In contrast to inducible gene expression systems for fundamental research, such systems for applications in the food industry should preferentially comprise components derived from host DNA and be regulated by a food-compatible effector. Thus far, only very few food-grade regulatable gene expression systems have been developed ${ }^{5-7}$. A system in which a change in temperature triggers gene expression would be useful as such a temperature shift constitutes an initial step in cheese manufacturing.

The most widely used system for temperature-controlled gene expression in bacteria is that developed for Escherichia coli on the basis of the bacteriophage lambda $(\lambda)$ immunity region in combination with a temperature-sensitive (Ts) CI repressor mutant, CI857 (refs. 8-10). CI belongs to a family of repressors that, despite significant differences in their amino-acid sequences, show very similar three-dimensional folding. They have a two-domain structure with an $\mathrm{N}$-terminal portion involved in DNA binding and a $\mathrm{C}$ terminal domain that mediates dimer formation, dimer-dimer interaction, and self-cleavage ${ }^{11}$. The 'hinge region' connecting both domains contains a conserved site that can undergo RecA-mediated autodigestion resulting in inactivation of the repressor ${ }^{12}$. Threedimensional structures of the N-terminal domains of several members of this repressor family are available. The $\mathrm{N}$-terminus is made up of $5 \alpha$ helices of which helix 2 and helix 3 (the helix-turn-helix motif) are involved in nucleotide sequence-specific DNA recognition $^{13}$. Helix 5 contributes to the hydrophobic packing at the interface of the monomers in the functional dimer ${ }^{24}$.

The immunity region of the temperate lactococcal bacteriophage r1t encompasses the gene specifying the regulator protein Rro and the operator sites to which Rro binds ${ }^{15}$. Although very limited similarities exist in the primary amino-acid sequences, Rro shares the characteristic two-domain structure of the $\lambda$ CI-like repressors. We use molecular modeling to build models of the N-terminus of Rro on the basis of the known $3 \mathrm{D}$-structures of the CI repressors of the $E$. coli bacteriophages $\lambda$ and 434 (refs. 16,17). The models were used to predict which amino acids to target in order to obtain variants of Rro with decreased stabilities at elevated temperatures. One of the mutants holds promise for temperature-inducible gene expression systems in L. lactis.

\section{Results and discussion}

Although several thermolabile $\lambda$ CI variants have been isolated ${ }^{18}$, mutants that are suitable for inducible gene expression systems are quite scarce. Such mutants should meet a number of requirements. At low temperatures they should retain wild-type properties, whereas when confronted with an elevated, nonpermissive temperature they should be unstable. The temperature range within which these changes should take place is rather narrow as the organism in which the system is to be used should survive at the induction temperature. The Ts derivative CI857 of the CI repressor carries only one amino-acid substitution in its $\mathrm{N}$-terminal domain and fulfills these requirements ${ }^{18}$. The replacement of $A 66$ by Thr resulted in a reduced stability of CI857 at elevated temperatures. In order to understand the cause of temperature sensitivity, the effect of the A66T substitution on the 3D-structure of the $\mathrm{N}$-terminal domain of CI was examined using the molecular modeling program WHAT IF $^{19}$. This analysis showed that A66 is buried within a densely packed hydrophobic pocket that comprises of several hydrophobic residues (Fig. 1A). Of these, L18 and F76 are completely buried in the core of the protein and have been shown to be critical for protein stability.0. Although the predicted contribution of the hydrophobicity of A66 to stability of the hydrophobic pocket is limited, substitution of the small and neutral side chain of Ala by the larger and more polar side chain of Thr is predicted to result in distortion of local hydrophobic interactions. This would agree with the earlier postulation of Hecht et al. ${ }^{21}$. A66T could also affect dimer stability as it is in close proximity to helix 5 at the interface between the two monomers. 
We decided to construct thermolabile derivatives of rlt repressor Rro on the basis of the CI857 modeling data. The 3D-structures of the $\mathrm{N}$-terminal domains of $\lambda \mathrm{CI}^{16}$ and $434 \mathrm{CI}^{17}$ were used as templates to build two independent models of the corresponding region of Rro. Although these proteins are members of the same family of repressors, homology in the amino-acid sequences of their N-termini is rather limited (Fig. 2). Comparison of predicted secondary structure elements, however, suggested considerable structural conservation. On the basis of the aligned amino acid sequences, the $\mathrm{N}$ terminal domain of Rro was modeled by structurally superimposing the $\mathrm{C} \alpha$ atoms of both Rro and $\lambda \mathrm{CI}$, and Rro and $434 \mathrm{CI}$ using WHAT IF. The models appeared to be very similar (Fig. 3 ).

The models obtained for the N-terminus of Rro showed that the core was mainly hydrophobic and most charged residues were at solvent-exposed positions, as is the case in the $N$-terminal region of $\lambda$ $\mathrm{CI}$ and $434 \mathrm{CI}$, suggesting that the models were faithful. Their fidelity was corroborated by the highly conserved packing in the hydrophobic pocket referred to above. In $\lambda \mathrm{Cl}$, the side chains of residues $\mathrm{A} 62$, L65, A66, L69, V73, and F76 strongly interact (Fig. 1A), whereas the Rro residues F50, M53, V54, F57, P61, and L64 are presumably

A

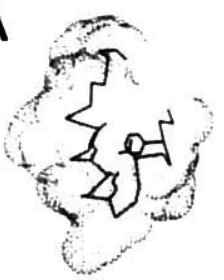

B
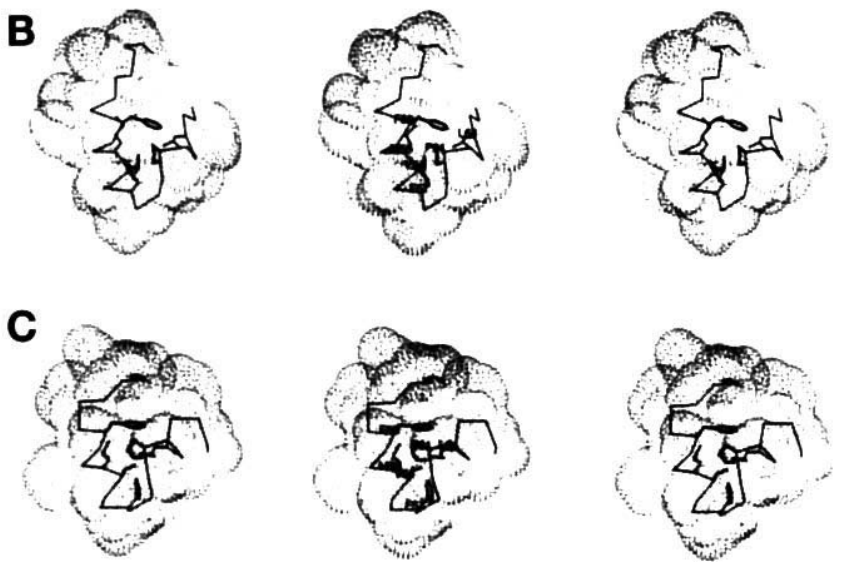

Figure 1. The hydrophobic pockets within the N-terminal domain of $\lambda \mathrm{Cl}$ (A) and the Rro models that were obtained using $\lambda \mathrm{Cl}$ (B) and $434 \mathrm{CI}$ (C) as templates. The hydrophobic core residues supposed to be important for their stability are indicated. The side chain of the $\lambda \mathrm{Cl}$ residue $\mathrm{A66}$, which has been shown to be critical for stability at elevated temperatures, and the side chains of the Rro residues that were randomized are depicted in bold. The solvent-accessible surfaces are dotted. involved in hydrophobic stabilization of a similar pocket (Fig. 1B and C). Interestingly, several conserved amino-acid interactions in CI and Rro were observed. For example, helix 4 residue $\mathrm{L} 69$ of $\mathrm{CI}$ has a strong hydrophobic interaction with $\mathrm{F} 51$ in helix 3 , which could be important for proper positioning of this DNA recognition helix. This interaction seems to be conserved in Rro via the predicted interaction between I39 (helix 3 ) and F57 (helix 4).

On the basis of the structural models of the hydrophobic pocket of Rro, it seems likely that replacement of residue V54which corresponds to the location of A66 in the hydrophobic pocket of $\lambda$ CI-by Thr would not dramatically affect the stability of Rro, as its side chain would be accommodated without major structural constraints (Fig. 1B and C).

To test this hypothesis and, thus, the validity of the models, Rro mutant V54T was constructed using polymerase chain reaction (PCR). The effect of the mutation on the thermostability of Rro was tested using plasmid pIR12 (ref. 15). As expression of lac $Z$ on this plasmid is repressed by the action of Rro, L. lactis cells carrying PIR 12 form white colonies on X-gal-containing agar plates. Colonies of cells harboring a thermolabile Rro mutant should stain blue at elevated temperatures. The stability of Rro at an elevated temperature $\left(40^{\circ} \mathrm{C}\right)$ was not affected by replacing V54 with Thr. As the model also suggests that P61 is important for the stability of the hydrophobic pocket of Rro, because it is located in the loop connecting helices 4 and 5 , this residue was replaced by either Ala or Gly. The resulting repressor variants were already nonfunctional at a low temperature $\left(24^{\circ} \mathrm{C}\right)$. Introduction of Val at this position, however, did not affect temperature sensitivity, suggesting that the destabilizing effects of Gly and Ala were not solely the result of increased local flexibility but, rather, were caused by a decrease of the hydrophobicity of the residue at position 61 .

The model of the hydrophobic pocket of Rro (Fig. $1 \mathrm{~B}$ and C) and the results obtained with the directed single mutants suggest that either one or a combination of the hydrophobic residues F50, V54, and P61 are important for its structural stability. In order to obtain a temperature-sensitive Rro variant that can be used for inducible gene expression purposes, these three positions were randomized using PCR. Mutagenized PCR fragments of the appropriate region in rro were used to replace the corresponding rro fragment in pIR12. The ligation mixture was used to transform $L$. lactis and cells were plated onto X-gal-containing agar plates which were incubated at room temperature. Of 1382 transformants, about $86 \%$ expressed a nonfunctional Rro mutant as their colonies were already blue at room temperature. The remaining (white) transformants were screened for lac $Z$ expression at $40^{\circ} \mathrm{C}$. Only 12 displayed a clearly higher level of lacZ expression at the elevated temperature. Plasmid INA was isolated from these strains and the mutated regions in rro were sequenced. In addition, lac $Z$ induction levels were quantified by measuring the $\beta$-galactosidase activities of the various strains after growth for three hours at $24^{\circ} \mathrm{C}$ and $40^{\circ} \mathrm{C}$.

All the mutants contained different amino-acid combinations at the randomized positions (Table 1). None contained other changes in the exchanged fragment of rro. Although there is still at least one hydrophobic residue present at one of the positions, the others were occupied with charged or polar residues, or with glycine. Instability at elevated temperatures of proteins in which hydrophobic buried residues had been replaced by nonhydrophobic amino acids has been observed before ${ }^{22}$. Residue 50 appears to be critical for obtaining the desired Ts phenotype, as only three amino acids (Leu, Trp, and Ser) were found at this position. On the basis of the specific residue at this position the variants can be differentiated into three classes with induction levels rang- 
A

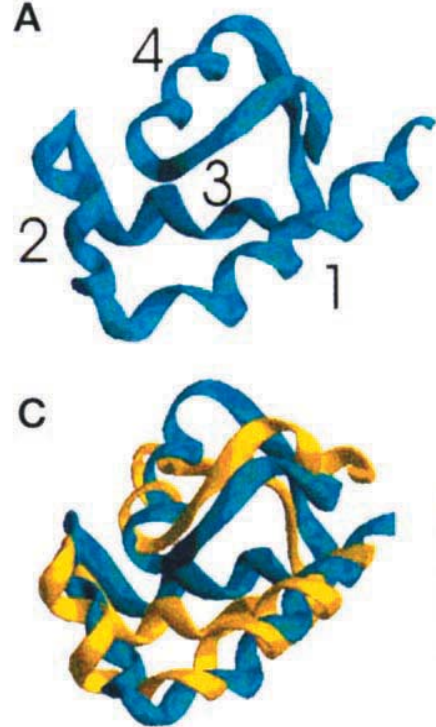

B

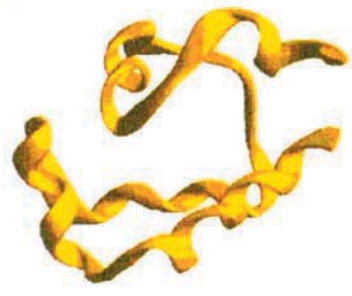

Figure 3. Ribbon models of the DNA binding $\mathrm{N}$-terminal domain of Rro using the 3D-structures of $\lambda \mathrm{Cl}(\mathrm{A}), 434 \mathrm{Cl}(\mathrm{B})$, and the superimposing of the two models (C). The numbers indicate the first four $\alpha$-helices.

Table 1. Characteristics of Rro and its thermosensitive mutants.

\begin{tabular}{|c|c|c|c|c|c|c|}
\hline $\begin{array}{l}\text { Rro/ } \\
\text { Mutant }\end{array}$ & $\begin{array}{l}\text { Am } \\
\text { po: }\end{array}$ & on & & $\begin{array}{l}\beta \text {-galactos } \\
\text { activity }\end{array}$ & & $\begin{array}{l}\text { Induction } \\
\text { level }^{\dagger}\end{array}$ \\
\hline & 50 & 54 & 61 & $24^{\circ} \mathrm{C}$ & $40^{\circ} \mathrm{C}$ & \\
\hline Rro & $\mathrm{F}$ & V & $P$ & $0.96 \pm 0.02$ & $1.14 \pm 0.01$ & $1.19 \pm 0.01^{\prime}$ \\
\hline 1 & $\mathrm{~L}$ & $N$ & $G$ & $0.82 \pm 0.01$ & $27.1 \pm 0.2$ & $33.0 \pm 0.7$ \\
\hline 2 & $L$ & $\mathbf{R}$ & $F$ & $0.71 \pm 0.02$ & $48.8 \pm 0.3$ & $68.8 \pm 1.2$ \\
\hline 3 & $L$ & $Q$ & $\mathrm{G}$ & $1.12 \pm 0.03$ & $81.9 \pm 0.6$ & $73.2 \pm 2.5$ \\
\hline 4 & $L$ & $\mathrm{~F}$ & $T$ & $0.92 \pm 0.02$ & $77.1 \pm 0.8$ & $83.8 \pm 1.3$ \\
\hline 5 & $L$ & $\mathrm{G}$ & $N$ & $1.44 \pm 0.01$ & $151.0 \pm 2.0$ & $105.0 \pm 1.0$ \\
\hline 6 & $W$ & $\mathrm{R}$ & V & $1.03 \pm 0.02$ & $50.1 \pm 0.5$ & $48.6 \pm 1.2$ \\
\hline 7 & W & $\mathrm{N}$ & I & $1.10 \pm 0.03$ & $151.0 \pm 3.0$ & $138.0 \pm 3.0$ \\
\hline 8 & W & A & $\mathrm{G}$ & $1.10 \pm 0.03$ & $172.0 \pm 2.0$ & $156.0 \pm 3.0$ \\
\hline 9 & W & $\mathrm{K}$ & $M$ & $1.09 \pm 0.01$ & $274.0 \pm 4.0$ & $252.0 \pm 3.0$ \\
\hline 10 & $S$ & $K$ & L & $1.15 \pm 0.03$ & $234.0 \pm 3.0$ & $204.0 \pm 4.0$ \\
\hline 11 & $\mathrm{~S}$ & A & W & $1.02 \pm 0.02$ & $363.0 \pm 4.0$ & $356.0 \pm 3.0$ \\
\hline 12 & $\mathrm{~S}$ & V & $A$ & $0.66 \pm 0.01$ & $392.0 \pm 3.0$ & $594.0 \pm 4.0$ \\
\hline
\end{tabular}

*Units for $\beta$-galactosidase activity are indicated in the Experimental protocol section. The induction level was obtained by dividing the $\beta$-galactosidase activity at $40^{\circ} \mathrm{C}$ by that observed at $24^{\circ} \mathrm{C}$. The values for the $\beta$-galactosidase activities represent the mean of three independent experiments. In all cases the standard errors did not exceed 3\%. *The induction level obtained from pIR12 after growth of the host strain for 3 hours at $24^{\circ} \mathrm{C}$ in the presence of $1 \mu \mathrm{g} \mathrm{ml}^{-1}$ mitomycin $\mathrm{C}$ was $542 \pm 4$.

ing from 33 to 105 (Leu), 49 to 252 (Trp), and 204 to 594 (Ser). Wildtype Rro contains the large and hydrophobic residue Phe at position 50. Its replacement by the smaller but hydrophobic residue Leu probably causes some distortion in the hydrophobic packing. This effect is expected to be more pronounced when F50 is replaced by the small and polar residue Ser. The observed intermediate Ts phenotypes of the variants that contain the large and slightly polar Trp residue at this position are also in agreement with the assumption that both the size and hydrophobicity of the residue at this position are important for temperature sensitivity. The gradation in thermosensitivity within the three classes indicates that the characteristics of the residues at positions 54 and 61 and the steric consequences of their accommodation also have an effect on the stability at elevated temperatures. For example, comparison of the induction levels observed for mutants Rrol and Rro3, which only differ by the presence of an Asn or Gln residue at position 54, respectively, shows that the size of the side chains at this position can have a profound effect on thermal stability. The importance of steric compatability of the introduced residues is
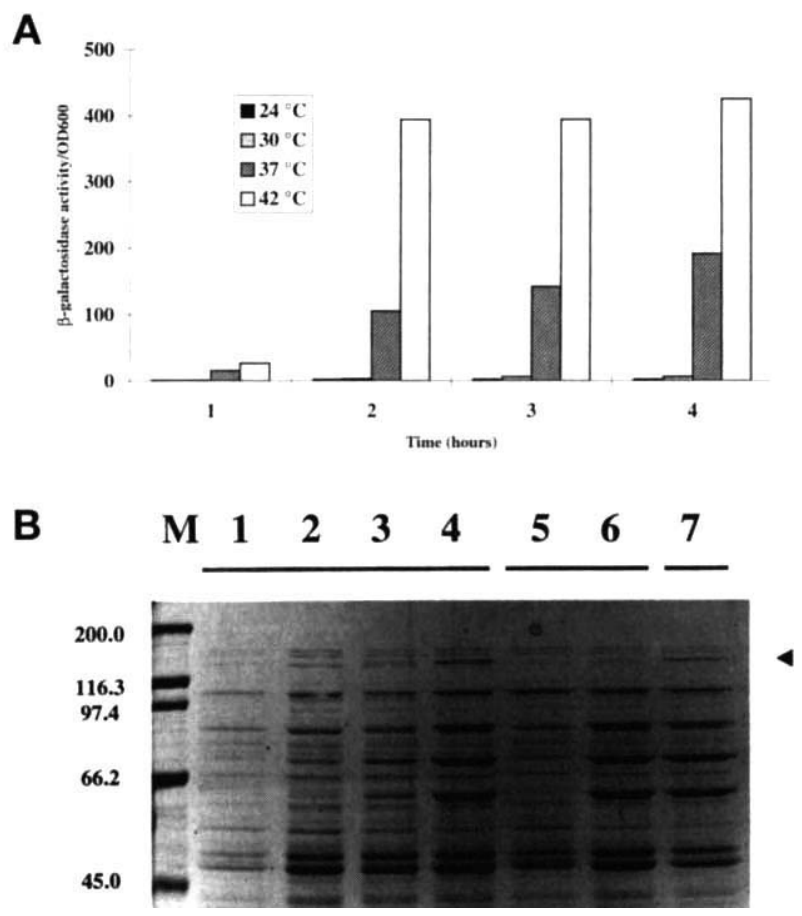

Figure 4. (A) Effect of growth of $L$ lactis at different temperatures on the $\beta$-galactosidase activities obtained with Rro12 as a function of time. Time indicated is postincubation. (B) Temperature-inchuced production of $\beta$-galactosidase. Cell-free extracts of cells expressing lac $Z$ under control of Rro12 were grown for 3 hours at 24 (lane 1), 30 (lane 2), 37 (lane 3), and $42^{\circ} \mathrm{C}$ (lane 4). Cell-free extracts of L. lactis (pIR12) grown at 24 (lane 5 ) and $42^{\circ} \mathrm{C}$ (lane 6) for 3 hours. Cell-free extracts of L. lactis (pIR13) that constitutively expresses lac Z, grown at $42^{\circ} \mathrm{C}$ (lane 7). M: reference proteins. The arrow indicates the position of $\beta$-galactosidase.

illustrated by the observation that substitution of P61A is only allowed when a mutation at position 50 occurs simultaneously. As referred to above, the single P61A mutation resulted in a protein that was unstable at $24^{\circ} \mathrm{C}$. Mutant Rrol2, which performs best in terms of limited leakage at low temperature and high induction levels at the elevated temperature, carries the amino-acid substitution F50S in addition to P61A. Because of the selection criteria used, it can not be determined whether other residues at position 50 can compensate for P61A, to the extent that a functional Rro variant could be obtained at the elevated temperature.

It appears that the level of induction obtainable with Rrol2 can be easily modulated by growing the cells at different (nonpermissive) temperatures (Fig. 4A) and can be as high as that obtained with mitomycin C using wild-type Rro (Table 1 ). At $30^{\circ} \mathrm{C}$, the temperature at which $L$. lactis is normally cultivated, $\beta$-galactosidase activity is low. The level obtained after three hours of growth at this temperature is about six times higher than the "uninduced" control (grown at $24^{\circ} \mathrm{C}$ ). Growth at $37^{\circ} \mathrm{C}$ results in a considerable increase in the $\beta$ galactosidase activity. Within the same period of time, the expression level had increased 290 -fold. The induction level at $42^{\circ} \mathrm{C}$ was more than twice that observed at $37^{\circ} \mathrm{C}$. The enzymatic activities in cell-free extracts correlated with the amount of intracellularly produced protein (Fig. 4B). In addition to bands representing several heat shock-induced proteins, a band at a position corresponding to that of $\beta$-galactosidase could be discerned after growth at $37^{\circ} \mathrm{C}$ and $42^{\circ} \mathrm{C}$. The specific features of Rro12 enable its use in systems with which the level of expression of a specific gene product can be modulated at any stage of growth of $L$. lactis simply by changing the temperature.

Although a procedure to predict residues in amino-acid sequences which, if mutated, would likely result in a Ts phenotype 
has recently been developed ${ }^{22}$, single-site mutations will often result in defective mutants or need to be compensated for by additional mutations to obtain a protein that is stable below the nonpermissive temperature. We demonstrate that conserved structura] features in nonhomologous but related proteins can be extremely helpful in the design of (Ts) mutants. The structural information of buried residues and their predicted stabilizing interactions was shown to enable the specific targeting of amino acids to be randomized in order to obtain the desired phenotype.

\section{Experimental protocol}

Bacterial strains, plasmids, and media. E. coli MC1000 (araD)139 4 lacx74 $\Delta($ ara, leu $) 7697$ galU galK str $A^{23}$ was grown in TY broth ${ }^{2 a}$ or on TY $(1.5 \%)$ agar plates. L. lactis LI.302 (MG1363 carrying the pWV01 repA gene on its chromosome $)^{25}$ was grown in glucose $1 / 2 \mathrm{M} 17$ broth $^{26}$, or on glucose $1 / 2 \mathrm{M} 17$ (1.5\%) agar plates. Erythromycin was used at 100 and $5 \mu \mathrm{g} / \mathrm{ml}$ for E. coli and L. lactis, respectively. X-gal (Sigma Chemical, St. Louis, MO) was added to plates at a final concentration of $40 \mathrm{~g} / \mathrm{ml}$. Plasmid pIR 12 harbors the regulatory region of $L$. lactis bacteriophage r1t encompassing rro, encoding the phage repressor, and tec, the topological equivalent of lambda cro. E. coli lacZ is translationally fused to ORF5 immediately downstream of $t e c^{35}$. Its derivative pIR13 carries a frameshift mutation within $r r^{15}$.

DNA techniques and transformation. Plasmid DNA was isolated by the method of Birnboim and Doly ${ }^{27}$. Restriction enzymes and T4 DNA ligase were obtained from Boehringer GmbH (Mannheim, Germany) and used according to the manufacturer's instructions. PCR was performed using the GeneAmp2400 system (Perkin Elmer, Norwalk, CT). Samples were heated to $94^{\circ} \mathrm{C}$ for $2 \mathrm{~min}$, after which target DNA was amplified in 25 cycles: $94^{\circ} \mathrm{C}$ for 1 min; $50^{\circ} \mathrm{C}$ for 2 $\mathrm{min}$; and $73^{\circ} \mathrm{C}$ for $1 \mathrm{~min}$. For nucleotide sequence analyses the dideoxynucleotide chain termination method ${ }^{24}$ was used with $\left[\alpha-{ }^{35} \mathrm{~S}\right]$-dATP (Amersham, Buckinghamshire, UK) and the T7 sequencing kit and protocol (Pharmacia, Uppsala, Sweden). Plasmids were introduced in L. lactis LL302 by electrotransformation ${ }^{25}$. E. coli was transformed by the method of Mandel and Higa ${ }^{28}$.

Molecular modeling. The program WHAT IF ${ }^{19}$ was used for model building, mutant prediction, and structural analysis. The 3D-structures of $\lambda \mathrm{CI}$ and 434 CI were obtained from the Protein Data Bank at Brookhaven. The PDB entry codes and their respective resolutions are $1 \mathrm{lmb}(1.8 \AA)$ and 1 rpe $(2.5 \AA)$, respectively. Sequence alignments were performed using the Clustal method of the MEGALIGN program of the DNASTAR software package (DNASTAR) Secondary structure predictions were performed according to Rost and Sander ${ }^{29}$.

Mutagenesis and mutant screening. Oligonucleotide-directed mutagenesis of ro was performed by PCR amplification of a unique $372 \mathrm{bp} \mathrm{Ncol/FclXI} \mathrm{frag-}$ ment (nucleotides 2624 to 2271 of the rlt sequence; EMBI./GenBank/DDB] Nucleotide Sequence Data Library Accession Number U38906) using Vent DNA polymerase (New England Biolábs, Beverly, MA). Mutation primers were synthesized on an Applied Biosystems 392A synthesizer (Applied Biosystem, Foster City, $\mathrm{CA}$ ) and either contained specific mutations to generate single-site mutations or had inosines incorporated at positions constituting the codons to be randomized. After digestion of the PCR products with $\mathrm{NcoI}$ and EclXI, the fragments were ligated into corresponding restriction enzyme sites of PIR12, thereby replacing the wild-type rro subfragment. 'The ligation mixture was used to electrotransform $L$. lactis LI.302. 'Transformants were plated on X-gal-containing glucose 1/2M17 plates and incubated at room temperature. White colonies were tested for the presence of a temperature sensitive repressor mutant by transferring them to two plates, one of which was incubated at $24^{\circ} \mathrm{C}$ and the other at $40^{\circ} \mathrm{C}$. Plasmid DNA was isolated from cells displaying temperature-dependent blue staining and introduced into E. coli by transformation. The plasmid DNA isolated from $E$. coli was used for nucleotide sequence analyses.

Temperature induction. Overnight cultures of $L$. lactis (grown at $24^{\circ} \mathrm{C}$ ) were diluted 100 -fold in fresh glucose $1 / 2 \mathrm{Ml} 7$ medium and grown at $24^{\circ} \mathrm{C}$ until the cultures had reached an $O D_{s \infty}$ of 0.3 . At that stage the cultures were divided into equal portions, which were incubated for 3 hours at various higher temperatures.

Preparation of cell-free extracts. Cells were collected by centrifugation at $20,000 \times \mathrm{G}$ for $1 \mathrm{~min}$ and resuspended in the appropriate buffer. Glass beads $\left(0.25-0.50 \mathrm{~mm}\right.$ in diameter) were added, and cells were disrupted at $4^{\circ} \mathrm{C}$ for 10 min using a "Shake it, Baby" cell disrupter (Biospec Products, Bartville, $O K)$. Glass beads and cells debris were removed by centrifugation for $6 \mathrm{~min}$ in an Eppendorf centrifuge.

Assay of $\beta$-galactosidase activity. Cells from $4 \mathrm{ml}$ of cultures were sedimented and subsequently resuspended in $1 \mathrm{ml}$ cold $Z$-buffer ${ }^{30}$, after which cell-free extracts were made. The $\beta$-galactosidase activity per $O D_{6 n 0}$ was determined essentially as described by Miller" ( 1 unit $=1000 \times \mathrm{OD}_{420} / \mathrm{sam}-$ ple volume (in $\mathrm{ml}) \times$ incubation time (in $\left.\mathrm{min}) \times \mathrm{OD}_{600}\right)$.

SDS-PAGE. Sodium dodecyl sulfate-polyacrylamide gel electrophoresis (SDSPAGE) was performed according to the protocol of Laemmli ${ }^{3}$, using the Protean II Minigel System (Bio-Rad Laboratories, Richmond, CA). Prior to loading on a $10 \%$ PAA gel, cell-free extracts (from $2 \mathrm{ml}$ of culture) were incubated at $100^{\circ} \mathrm{C}$ for $1 \mathrm{~min}$. The prestained molecular-weight marker was obtained from Bio-Rad. SDS-polyacrylamide gels were stained with Coomassie brilliant blue.

\section{Acknowledgments}

We thank Unilever Research Laboratory, Vlaardingen, The Netherlands, for financial support and Aat Ledeboer, Wouter Musters, and Gert Vriend for helpful discussions. JK is the recipient of a fellowship of the Royal Netherlands Acadamy of Arts and Sciences (KNAW).

1. Kok, J. 1993. Genetics of proteolytic enzymes of lactococci and their role in cheese flavor development. J. Dairy. Sci. 76:2056-2064.

2. Visser, S. 1993. Proteolytic enzymes and their relation to cheese ripening and flavor: an overview. J. Dairy. Sci. 76:329-350.

3. Crow, V.L, Coolbear, T., Gopal, P.K., Martley, F.G., McKay, L.L., and Riepe, H. 1995. The role of autolysis of lactic acid bacteria in the ripening of cheese. Int. Dairy. J. 5:855-875.

4. Fox, P.F., Wallace, J.M., Morgan, S., Lynch, C.M., Niland, E.J., and Tobin, J. 1996. Acceleration of cheese ripening. Antonie van Leeuwenhoek 70:271-297.

5. Van Roolien, R.J., Gasson, M.J., and de Vos, W.M. 1992. Characterization of thepromoter of the Lactococcus lactis lactose operon: contribution of flanking sequences and LacR repressor to its activity. J. Bacteriol. 174:2273-2280.

6. de Ruyter, P.G.G.A., Kuipers, O.P., and de Vos, W.M. 1996. Controlled gene expression systems for Lactococcus lactis with the food-grade inducer nisin Appl. Environ. Microbiol. 62:3662-3667.

7. O'Sullivan, D.J., Walker, S.A., West, S.G., and Klaenhammer, T.R. 1996 Development of an explosive expression strategy using a lytic phage to trigger explosive plasmid amplification and gene expression. Bio/Technology 14:82-87.

8. Hendrix, R.W., Roberts, J.W., Stahl, F.W., and Weisberg, R.A. (eds.) 1983 Lambda II. Cold Spring Harbor Laboratory Press, Cold Spring Harbor, NY.

9. Rosenberg, M., Ho, Y.-S., and Shatzman, A.R. 1983. The use of pKC30 and its derivatives for controlled expression of genes. Methods Enzymol. 101:123-138.

10. Shatzman, A.R., and Rosenberg, M. 1986. Efficient expression of heterologous genes in Escherichia coli: the pAS vector system and its applications. Ann. N.Y. Acad. Sci. 476:233-248.

11. Pabo, C.O., Sauer, R.T., Sturtevant, J.M., and Ptashne, M. 1979. The repressor contains two domains. Proc. Natl. Acad. Sci. USA 76:1608-1612.

12. Little, R.J. 1993. LexA cleavage and other self-processing reactions. J. Bacteriol 175:4943-4950.

13. Pabo, C.O. and Saver, R.T. 1992. Transcription factors: structural families and principles of DNA recognition. Annu. Rev. Biochem. 61:1053-1095.

14. Pabo, C.O. and Lewis, M. 1982. The operator-binding domain of repressor: structure and DNA recognition. Nature 298:443-447.

15. Nauta, A., van Sinderen, D., Karsens, H., Venema, G., and Kok, J. 1996. Inducible gene expression mediated by a repressor-operator system isolated from Lactococcus lactis bacteriophage r1t. Mol. Microbiol. 19:1331-1341.

16. Beamer, L.J. and Pabo, C.O. 1992. Refined $1.8 \AA$ crystal structure of the lambda repressor-operator complex. J. Mol. Biol. 227:1777-196.

17. Aggarwal, A.K., Rodgers, D.W., Drottar, M., Ptashne, M., and Harrison, S.C. 1988. Recognition of a DNA operator by the repressor of phage 434: A view at high resolution. Science 242:899-907.

18. Lieb, M. 1981. A fine structure map of spontaneous and induced mutations in the lambda repressor gene, including insertions of IS elements. Mol. Gen. Genet. 184:364-371.

19. Vriend, G. 1990. WHAT IF, a molecular modeling and drug design program. J. Mol. Graph. 8:52-56.

20. Reidhaar-Oison, J.F. and Sauer, R.T. 1990. Functionally acceptable substitutions in two $\alpha$-helical regions of $\lambda$ repressor. Proteins 7:306-316

21. Hecht, M.H., Sturtevant, J.M., and Sauer, R.T. 1984. Effect of single amino acid replacements on the thermal stability of the $\mathrm{NH}_{2}$-terminal domain of phage $\lambda$ repressor. Proc. Natt. Acad. Sci. USA 81:5658-5689.

22. Varadarajan, R., Nagarajaram, H.A., and Ramakrishnan, C. 1996. A procedure for the prediction of temperature-sensitive mutants of a globular protein based solely on the amino acid sequence. Proc. Natl. Acad. Sci. USA 93:13908-13913.

23. Casadaban, M.J. and Cohen, S.N. 1980. Analysis of gene control signals by DNA fusion and cloning in Escherichia coll. J. Mol. Biol. 138:179-207.

24. Rottlander, E. and Trautner, T.A. 1970. Genetic and transfection studies with Bacillus subtilis phage SP50. J. Mol. Biol. 108:47-60.

25. Leenhouts, K.J. and Venema, G. 1993. Lactococcal plasmid vectors. pp. 65-94 in Plasmids, A Practical Approach. Hardy, K.G. (ed.) Oxford University Press, Oxford, UK.

26. Terzaghi, B.E. and Sandine, W.E. 1975, Improved medium for lactic streptococci and their bacteriophages. Appl. Microbiol. 29:807-813.

27. Birnboim, H.C and Doly, J. 1979. A rapid alkaline extraction procedure for screening recombinant plasmid DNA. Nucleic Acids. Res. 7:1513-1523.

28. Mandel, M. and Higa, A. 1970. Calcium-dependent bacteriophage DNA infection. J. Mol. Biol. 53:159-162.

29. Rost, B. and Sander, C. 1993. Prediction of protein secondary structure at better than $70 \%$ accuracy. J. Mol. Biol. 232:584-599.

30. Miller, J. 1972. Experiments in Molecular Genetics. Cold Spring Harbor Laboratory Press, Coid Spring Marbor, NY.

31. Laemmli, U.K. 1979. Cleavage of structural proteins during the assembly of the head of bacteriophage T4. Nature 227:680-685. 